\title{
Methylammonium Governs Structural and Optical Properties of Hybrid Lead Halide Perovskites through Dynamic Hydrogen Bonding
}

\author{
Gabriele Saleh $^{1 *}$, Giulia Biffi ${ }^{1,2,3}$, Francesco Di Stasio ${ }^{1}$, , Beatriz Martín-García ${ }^{1,4}$, Ahmed L. Abdelhady ${ }^{1}$, \\ Liberato Manna ${ }^{1}$, Roman Krahne ${ }^{1}$, Sergey Artyukhin ${ }^{1}$ \\ ${ }^{1}$ Istituto Italiano di Tecnologia, Via Morego 30, 16163 Genova, Italy \\ ${ }^{2}$ Dipartimento di Chimica e Chimica Industriale, Università degli Studi di Genova, Via Dodecaneso 31, \\ 16146 Genova, Italy \\ ${ }^{3}$ Materials Physics Center, Spanish National Research Council, Paseo Manuel de Lardizabal 5, Donostia- \\ San Sebastián 20018, Gipuzkoa, Spain \\ ${ }^{4}$ CIC nanoGUNE, Tolosa Hiribidea, 76, 20018 Donostia-San Sebastian, Spain \\ *email: gabrielesaleh@outlook.com
}

\section{Supporting Information}




\section{Contents}

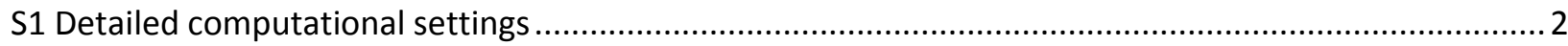

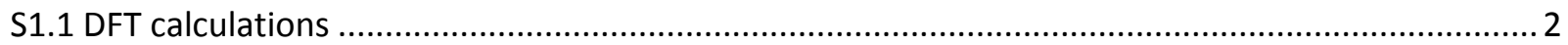

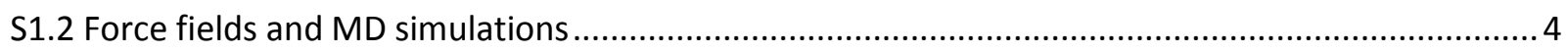

S1.3 Pearson correlation coefficient and other dipole-related quantities from MD trajectories. ........... 5

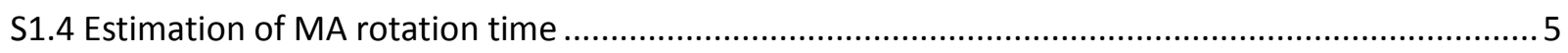

S2 Statistical quantities calculated from MD trajectories and results of energy and band gap fitting........ 7

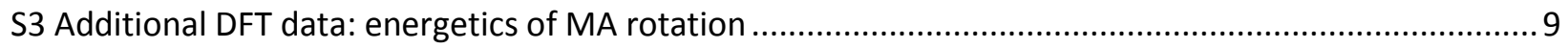

S4 Additional DFT data: density of states, charge density plots, and orbital overlap ............................. 12

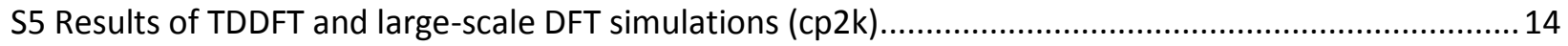

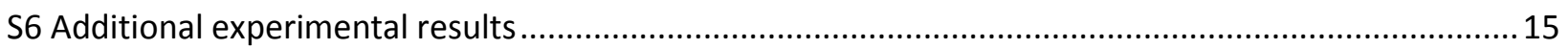




\section{S1 Detailed computational settings}

\section{S1.1 DFT calculations}

DFT calculations, general settings. When not otherwise stated, the following settings were adopted for the calculations performed with the VASP code $^{1}$. All the calculations were carried out with Density Functional Theory (DFT) Hamiltonians in the framework of the all-electron projector augmented wave (PAW) method ${ }^{2}$. The PAW pseudopotentials used were those recommended by the VASP website ${ }^{3}$. The PBE exchange-correlation functional was adopted ${ }^{4}$. The Tktchenko-Scheffler ${ }^{5}$ (TS) correction for dispersion was applied, since it was shown to give accurate results on these materials at a modest computational $\operatorname{cost}^{6}$. The plane wave kinetic energy cutoff was set to $400 \mathrm{eV}$. The Self Consistent Field procedure was considered converged when the energy difference between two consecutive cycles was lower than $1 * 10^{-6} \mathrm{eV}\left(1 * 10^{-5} \mathrm{eV}\right.$ for slabs), while structure optimization runs were stopped when the energy difference between one step and the previous one was lower than $1 * 10^{-5} \mathrm{eV}\left(1 * 10^{-4} \mathrm{eV}\right.$ for slabs). Note that Spin-Orbit Coupling (SOC), known to affect the band gap, was neglected. Nonetheless, our calculated values for the band gap (2.1 eV for the ground state) were close the experimental estimate $\left(2.21 \mathrm{eV}^{7}\right)$. This is because of the error compensation between the band gap underestimation of GGA functionals such as PBE, and the overestimation brought about by the neglect of SOC. While this error cancellation cannot be relied upon when trying to predict band gaps of new material, it is accurate enough to study the qualitative changes of band gaps such as those investigated in this study. In particular, it allowed to sample a high number of MA configurations and to perform simple DFT calculations of a large cell (in cp2k, see below), which would not have been possible were a hybrid functional and SOC correction adopted.

2x2x2 supercells sampling MA configurations (Fig. 1 of the main text). The initial geometries of the 110 sampled MA configurations were generated as follows. 74 were simply snapshots of plain force fields MD runs at various temperatures from 350 to $500 \mathrm{~K}$ (see below for lammps settings). 7 structures, including the ground state, were obtained from various simulated annealing MD runs, where the simulation box was heated to $\mathrm{T}=400 \mathrm{~K}$, let at $400 \mathrm{~K}$ for about $1 \mathrm{~ns}$, and then cooled down to $\mathrm{T}=30 \mathrm{~K}$, repeatedly. Other 20 structures were generated through a similar annealing, but constraining the MA molecules to have the $\mathrm{CN}$ axes lying in the same plane along $z$, similarly to the ground state. This was done once it was noticed, from the results of the MD runs described above, that some of these 'coplanar' configurations are particularly low in energy. The remaining 9 structures were generated by hand to sample some particular geometries (centrosymmetric, maximally ferroelectric, etc.). For each of those 110 structures, a DFT calculation was performed, relaxing all atomic positions and unit cell parameters to the closest minimum energy value. The reciprocal space was sampled through a $2 \times 2 \times 2$ uniform, $\Gamma$-centered grid. Note that it was checked that in the final set there are no structures that are identical to one another, i.e. 110 distinct local minima were sampled.

Density of states. The density of states was calculated on the minimum energy geometry with the same settings as above except that the reciprocal space was sampled with a finer, $3 \times 3 \times 3$ grid. For atomic projections, given the central role of $\mathrm{Pb}$ and $\mathrm{Br}$ for the optical properties, we changed their default radii to the estimate for ionic radii of $\mathrm{Pb}^{2+}$ and $\mathrm{Br}^{-}$, namely 1.33 and $1.82 \AA$. We note however, that default radii produce the same qualitative features as those we adopted. 
Surfaces simulated with DFT. 001 surfaces were simulated as 5-layers thick slabs with a $2 \times 2$ unit cell in the two directions parallel to the slab (Fig. S6). Periodic images along the $z$ direction were separated by 9 $\AA$ Af vacuum. The reciprocal space was sampled with a $2 \times 2 \times 1$ grid. The unit cell was frozen, using the bulk value for the directions parallel to the slab, while atomic positions were fully relaxed. The bulk reference calculations (Fig. S6) were performed on a $2 \times 2 \times 4$ supercell (192 atoms), with the same k-space grid as surfaces. The starting geometries for Fig. $\mathrm{S} 6$ were created by manually changing the atomic coordinates in the input file and verifying the resulting geometry by visualizing the structure.

Nudged Elastic Band calculations (energy barrier for MA rotation). Climbing-Image, Variable-Cell NEB (ClVC-NEB) calculations were performed by generating 3 intermediate structures between the ground state and the same structure having one molecule rotated by $180^{\circ}$ around the $\mathrm{c}$ axis (see Fig. S3; the final geometry was relaxed, too). The 'climbing image' method ${ }^{8}$ is chosen to ensure that one of the images is the saddle point connecting reactant and product, thereby having an accurate estimation of the reaction barrier. The spring constant among images was set to $5.0 \mathrm{eV} / \AA^{2}$, and the NEB convergence threshold was set to $1 * 10^{-4} \mathrm{eV}$.

Large calculations with cp2k. Calculations on large cells (4x4x16, 3072 atoms) and TDDFT calculations were performed through the $\mathrm{cp} 2 \mathrm{k}$ code ${ }^{9}$. A double zeta plus polarization basis set (DZVP) was adopted for all atoms, and the plane wave cutoff was set to 200 Ry and 125 Ry for TDDFT and large cell calculations, respectively. The SCF is considered converged when the accuracy (as calculated by cp $2 k$ ) is lower than $1 * 10^{-6} \mathrm{Ry}$, while for the geometry optimization the default convergence criteria are adopted ${ }^{10}$. The SCF procedure was carried out with the 'orbital transformation' algorithm, except for orbital energy (and band gap) calculations, for which the 'filter matrix' diagonalization algorithm was adopted. Cp2k is built a gamma-point only code, hence reciprocal space sampling is not possible. $4 \times 4 \times 16$ cells are large enough to expect bands to be flat: they would have been gamma-only also with the reciprocal space sampling criteria we adopted in VASP. TDDFT calculations were performed on $2 \times 2 \times 2$ cells from VASP (no geometry optimization), that were doubled in all 3 dimensions (thus becoming $4 \times 4 \times 4$ cells), so as to mimic the $2 \times 2 \times 2$ k-points net adopted in VASP. All these calculations are fully periodic.

Fittings. The fittings of eqs. 1-3 (main text) and Table S1 were carried out with a purposedly written python code which exploits the curve_fit function of scipy. The structural parameters needed for the fitting (bond lengths, angles, etc.) were calculated on the DFT optimized structure with the help of pymatgen library (https://pymatgen.org/). For each fitting, a set of different starting parameters were tested, and the ones that gave the best Pearson coefficient $R^{2}$ were adopted. Further tests to check the reliability of eqs. 1-3 were performed, i.e.:

- Eq. 1 contains a large number of fitting parameters, hence it could be argued that the good $\mathrm{R}^{2}$ obtained in the fitting stems from the large flexibility of the model and not from the physical basis discussed in the main text, namely that the energetics of MA configurations is determined by the HB network. To check this, we have broken the correspondence between the energy of each configuration and the number of $\mathrm{HBs}$ on each $\mathrm{Br}$ of the configuration ( $\mathrm{N}^{\mathrm{Br}}$ s of eq. 1 ) and performed the same fitting as in the original set of data. In practice, we have randomly reshuffled the energies so as to create a new dataset in which the energy of a given configuration is not anymore associated to its set of $\mathrm{N}^{\mathrm{Br}}$, but rather to a set of $\mathrm{N}^{\mathrm{Br}}$ belonging to another, randomly chosen, configuration. We have produced 10 of those randomly reshuffled datasets, on which we have performed the energy vs $\mathrm{N}^{\mathrm{Br}}$ fitting adopting eq. 1 . The $\mathrm{R}^{2}$ of those randomly reshuffled datasets ranged from 0.06 
to 0.16 (while $R^{2}=0.71$ with the actual dataset). This proves that the hypothesis discussed in the main text, namely that the energetics of MA configurations is largely determined by how the various $\mathrm{HBs}$ are distributed among $\mathrm{Br}$ acceptor atoms, is correct.

- In eq. 2, the third term treat both $\mathrm{N}-\mathrm{H} \cdots \mathrm{Br}$ and $\mathrm{C}-\mathrm{H} \cdots \mathrm{Br}$ on the same footing, as the same set of parameters ( $\mathrm{k} 3$ and $\mathrm{k} 4$ ) is adopted for all $\mathrm{HBs}$. We have tried to perform a fitting with different parameters for $\mathrm{N}-\mathrm{H} \cdots \mathrm{Br}$ and $\mathrm{C}-\mathrm{H} \cdots \mathrm{Br}$, in order to check whether treating all $\mathrm{HBs}$ with the same exponential dependence is a good approximation. That is, we fitted the energy of the MA configurations with the following equation:

$$
\begin{aligned}
& E=k_{1}+k_{2} \sum_{i}^{N_{B r}}\left[\pi-(P b \widehat{B r} P b)_{i}\right]^{2}+k_{3} \sum_{i}^{N_{C-H} \ldots B r} e^{-k_{4} d(H \cdots B r)_{i}}+ \\
& k^{\prime}{ }_{3} \sum_{i}^{N_{N-H \ldots B B r}} e^{-k^{\prime}{ }_{4} d(H \cdots B r)_{i}}+k_{5}\left\{\frac{\sum_{j}^{\left.N_{P b-B r}\left[d(P b B r)_{j}-\overline{d(P b B r}\right)\right]^{2}}}{N_{P b-B r}-1}\right\} .
\end{aligned}
$$

The resulting $R^{2}$ is not significantly improved with respect to eq. 2 of the main text (from 0.821 to 0.835 ), despite the considerably higher number of parameters adopted (from 5 to 7 ). This indicates that adopting the same set of parameters for all HBs is a reasonable approximation.

- Eq. 3 fits the band gaps adopting only parameters related to the $\mathrm{PbBr}$ scaffold, as our electronic structure investigation suggests that HBs do not influence the band gap directly, but only through a deformation of the $\mathrm{PbBr}$ sublattice. To prove this hypothesis, we have performed the same fitting as in eq. 3 adding a term that takes into account the HBs strength, i.e. we fitted the band gaps with the following equation:

$E g=k_{1}+k_{2}(\pi-\overline{P b \widehat{B r} P b})^{2}+k_{3} d(P b B r)_{\max }{ }^{2}+k_{4} \overline{d_{a v}(H \cdots B r)}$,

where $\mathrm{d}_{\mathrm{av}}(\mathrm{H} \cdots \mathrm{Br})$ represents the $\mathrm{H} \cdots \mathrm{Br}$ distance averaged over all $\mathrm{HBs}$ of a given $2 \times 2 \times 2$ cell. Despite increasing the number of fitting parameters from 3 to 4 , the $R^{2}$ hardly improves (from 0.730 to 0.733). This further supports the idea that HBs do not directly influence the band gap.

\section{S1.2 Force fields and MD simulations}

The MYP force field was adopted ${ }^{11}$. Note that, even though this force field was optimized for bulk simulations, it produced for surfaces the same minimum-energy structure as DFT, hence we deem it accurate enough for our qualitative investigation of MA dynamics. We believe that the reason for the good agreement is due to the fact that the surface effect on MA configurations is mainly determined by $\mathrm{HBs}$ (see main text), whose energetics is fairly well reproduced by force fields. The time step was $0.4 \mathrm{fs}$, and the Coulomb interactions were calculated through Ewald or pppm summations with an accuracy of $1 * 10^{-4} \mathrm{Kcal} / \mathrm{mole} / \AA ̊$ on forces. Nose-Hoover thermostats and barostats were adopted, with damping times of 400 and $4000 \mathrm{fs}$, respectively. Bulk calculations were performed on a 16x16x16 cell (49152 atoms) in a npt ensamble ( $p=1 \mathrm{~atm})$. Surface calculations were performed in an nVT ensamble (periodicity in the directions parallel to the slab taken from bulk calculations) on a 40-layer slab with a 8x8 periodicity in the two dimensions along the slab (30720 atoms), and no periodicity along the perpendicular direction. In both cases, the MD was let run for $2 \mathrm{~ns}$ after a $0.4 \mathrm{~ns}$ equilibration (during the first $0.1 \mathrm{~ns}$, the temperature was raised from $0 \mathrm{~K}$ to the desired temperature). The simulations exploited for creating the snapshots for cp2k band gap calculations were performed on a $4 \times 4 \times 16$ supercell (3072 atoms) in a nVT ensamble (volume fixed at ground state estimation). They were run for $25 \mathrm{~ns}$. 


\section{S1.3 Pearson correlation coefficient and other dipole-related quantities from MD}

\section{trajectories.}

The dipoles were calculated considering only $\mathrm{C}$ and $\mathrm{N}$ atoms of each molecule and they were made position independent by subtracting the geometric centre times the net charge of the $\mathrm{CN}$ fragment ("geometry" charge correction option of LAMMPS ${ }^{12}$ ). The resulting vector is basically parallel to the C-N axis. The calculations of dipole-related quantities (Pearson correlation coefficient, $\theta$ angle variance, etc.) were carried out with purposely built fortran codes, available upon request. The considered snapshots were 1 every 1000 for the whole duration of the MD except the equilibration time.

The Pearson correlation coefficient $\rho$ between two quantities $X$ and $Y$ measured is defined as:

$\rho_{X, Y}=\frac{\operatorname{cov}(X, Y)}{\sigma_{X} \sigma_{Y}}=\frac{\overline{\overline{\sum_{l}\left(X_{l}-\bar{X}\right)\left(Y_{l}-\bar{Y}\right)}}}{\sqrt{\sum_{i}\left(X_{i}-\bar{X}\right)^{2}} \sqrt{\sum_{i}\left(Y_{i}-\bar{Y}^{2}\right.}}$

where "cov" indicates the covariance and $\sigma$ the standard deviations. The overbar represents the average of a given quantity over the number of measurements $n$, over which the $i$ summation runs. In our case, we are interested in the Pearson correlation coefficient between two CN dipoles at a given distance, calculated over their distribution during an MD simulation. Therefore, the summation $i$ runs over the $n$ snapshots of the MD simulation. Since dipoles are vectors, the covariance between two dipoles $\mu_{1}$ and $\mu_{2}$ becomes a matrix ("cross-covariance matrix"), whose elements are:

$$
C_{a, b}\left(\mu_{1}, \mu_{2}\right)=\frac{1}{N-1} \sum_{i=1}^{N \operatorname{snap}}\left(\mu_{1, i}^{a}-\overline{\mu_{1, l}^{a}}\right)\left(\mu_{2, i}^{b}-\overline{\mu_{2, l}^{b}}\right)
$$

Here, $a$ and $b$ represent the Cartesian directions ( $x, y$, or $z)$, and their use as a superscript indicates the corresponding component of the dipole vector. Therefore, we calculated one Pearson correlation coefficient for each of the 9 elements of the cross covariance matrix (which is not necessarily symmetric) as:

$\rho^{a, b}\left(\mu_{1}, \mu_{2} ; r_{12}\right)=\frac{\sum_{i=1}^{N \operatorname{snap}}\left(\mu_{1, i}^{a}-\overline{\mu_{1, l}^{a}}\right)\left(\mu_{2, i}^{b}-\overline{\mu_{2, l}^{b}}\right)}{\sqrt{\sum_{i=1}^{N \operatorname{snap}}\left(\mu_{1, i}^{a}-\overline{\mu_{1, l}^{a}}\right)^{2}} \sqrt{\sum_{i=1}^{N \operatorname{snap}}\left(\mu_{2, i}^{b}-\overline{\left.\mu_{2, l}^{b}\right)^{2}}\right.}}$

Note that we added the $r_{12}$ dependence to highlight that this Pearson coefficient depends implicitly on the distance between dipoles. Finally, we group together all dipoles pairs whose centre of mass is separated by a given distance $R_{12}$ within a tolerance of $0.2 \AA$. Since the Pearson coefficient can be positive or negative (hence they would cancel out if normally averaged), while we are interested in its magnitude only, the average is performed over absolute values:

$\rho^{a, b}\left(R_{12}\right)=\frac{1}{N_{R 12}} \sum_{i, j}^{N \text { pairs }}\left|\rho^{a, b}\left(\mu_{i}, \mu_{j} ; r_{12}\right)\right|\left[H\left(R_{12}-r_{12}+0.2\right)-H\left(R_{12}-r_{12}-0.2\right)\right]$

where $H$ is the Heaviside step function and $N_{R 12}$ is the number of dipole pairs separated by a distance $R_{12}$. Note that the term in square parenthesis is just a formal expression to indicate that only those dipoles having a distance $r_{12}$ of $R_{12} \pm 0.2 \AA$ are included in the summation.

\section{S1.4 Estimation of MA rotation time}

In order to estimate the time a MA molecule takes to rotate at a given temperature, given the energy barrier for rotation, we start from the equation for the rate constant $\mathrm{K}$ derived by Herzfeld ${ }^{13}$ 
$K=\left[\frac{k T}{h}\left(1-e^{-\frac{h v}{k T}}\right)\right] e^{-\frac{\Delta E^{*}}{k T}}$

where $\mathrm{k}$ and $\mathrm{h}$ are the Boltzmann and Planck constant, respectively, and $\mathrm{T}$ is the temperature. $\mathrm{v}$ is the frequency of vibration in the direction of the 'reaction', which in our case means the vibration of the MA molecule in the direction of rotation (see below). $\Delta \mathrm{E}^{\neq}$represents the energy difference between the transition state and the starting state. This equation was developed for the dissociation of a diatomic molecule by applying statistical mechanics and collision theory. However, it can be exploited to roughly estimate the rate constant for MA rotation without performing expensive phonon calculations. In fact, the term in square parenthesis can be seen as an approximation to the vibrational entropy term in the Vineyard ${ }^{14}$ equation for ions migration in solids, on the line of the arguments adopted by Eyring ${ }^{15}$. The vibrational mode of interest for the molecular rotation is the one in which $\mathrm{CN}$ rigidly moves around its midpoint (named "nodding donkey" mode in ref. 16). Vibrational modes of $\mathrm{MAPbBr}_{3}$ were analyzed in details in ref. 16, and the 'nodding donkey' rotation was shown to have frequency of $138-151 \mathrm{~cm}^{-1}$ (modes 16 and 18). We use the average between those two values (transformed in hertz) to estimate $K$ of equation $\mathrm{S} 5$, and $1 / \mathrm{K}$, that is the time that it takes on average for a MA molecule to rotate. With the barrier calculated from NEB (Fig. S3), at $\mathrm{T}=300 \mathrm{~K}$, we obtain $1 / \mathrm{K}=41 \mathrm{~ns}$. If we use a value of $0.1 \mathrm{eV}$ for $\Delta \mathrm{E}^{ \pm}$, we obtain $1 / K=15.3 \mathrm{ps}$, a value in line with experimental estimates (see main text). 
S2 Statistical quantities calculated from MD trajectories and results of energy and band gap fitting.
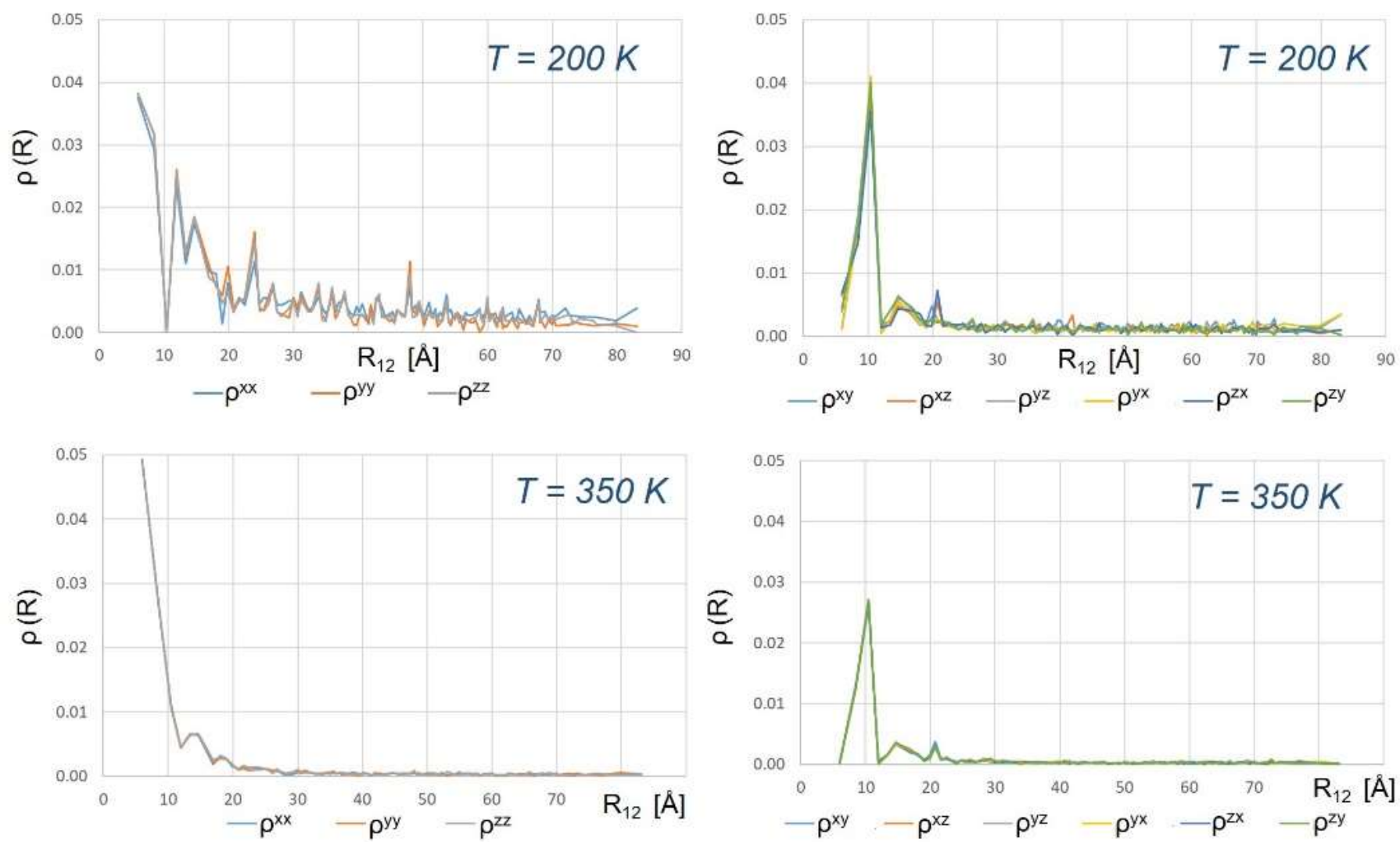

Figure S1. Average Pearson correlation coefficient (equation S4) between pairs of molecules as a function of the distance between them. Diagonal and off-diagonal elements are reported in different plots. Results obtained at two different temperatures are reported on the two different rows. 
Table S1 Results of the fitting of equations 1-3 of the main text.

\begin{tabular}{|c|c|c|c|c|}
\hline Eq. & Parameters & units & Standard Error ${ }^{a}$ & $\begin{array}{l}\text { sum of squared errors (meV/molecule); } \\
\mathrm{R}^{2}\end{array}$ \\
\hline 1 & $\begin{array}{l}1.8763 \\
1.2631 \\
-0.1568 \\
3.4262 \\
3.6481 \\
1.8822 \\
1.3755 \\
2.4690 \\
-2.4551 \\
1.0933\end{array}$ & $\begin{array}{l}\mathrm{meV} \\
\mathrm{meV} \\
\mathrm{meV} \\
\mathrm{meV} \\
\mathrm{meV} \\
\mathrm{meV} \\
\mathrm{meV} \\
\mathrm{meV} \\
\mathrm{meV} \\
\mathrm{meV}\end{array}$ & $\begin{array}{l}0.023 \\
0.093 \\
0.057 \\
0.098 \\
0.014 \\
0.376 \\
0.109 \\
0.086 \\
0.546 \\
0.087\end{array}$ & $8332 ; 0.7065$ \\
\hline 2 & $\begin{array}{l}146.43 \\
-4.7247 \\
-471342 \\
4.9861 \\
3560.6\end{array}$ & $\begin{array}{l}\mathrm{meV} \\
\mathrm{meV} / \mathrm{rad}^{2} \\
\mathrm{meV} \\
\AA^{-1} \\
\mathrm{meV} / \AA^{2}\end{array}$ & $\begin{array}{l}1.135 \\
0.210 \\
119146 \\
0.111 \\
217.1 \\
\end{array}$ & $5095 ; 0.8206$ \\
\hline 3 & $\begin{array}{l}0.2311 \\
1.710 \\
0.1656\end{array}$ & $\begin{array}{l}\mathrm{eV} \\
\mathrm{eV} / \mathrm{rad}^{2} \\
\mathrm{eV} / \AA^{2}\end{array}$ & $\begin{array}{l}6.73 \\
2.47 \\
0.706\end{array}$ & $0.1804 ; 0.7301$ \\
\hline
\end{tabular}

a Square root of the diagonal elements of the variance-covariance matrix

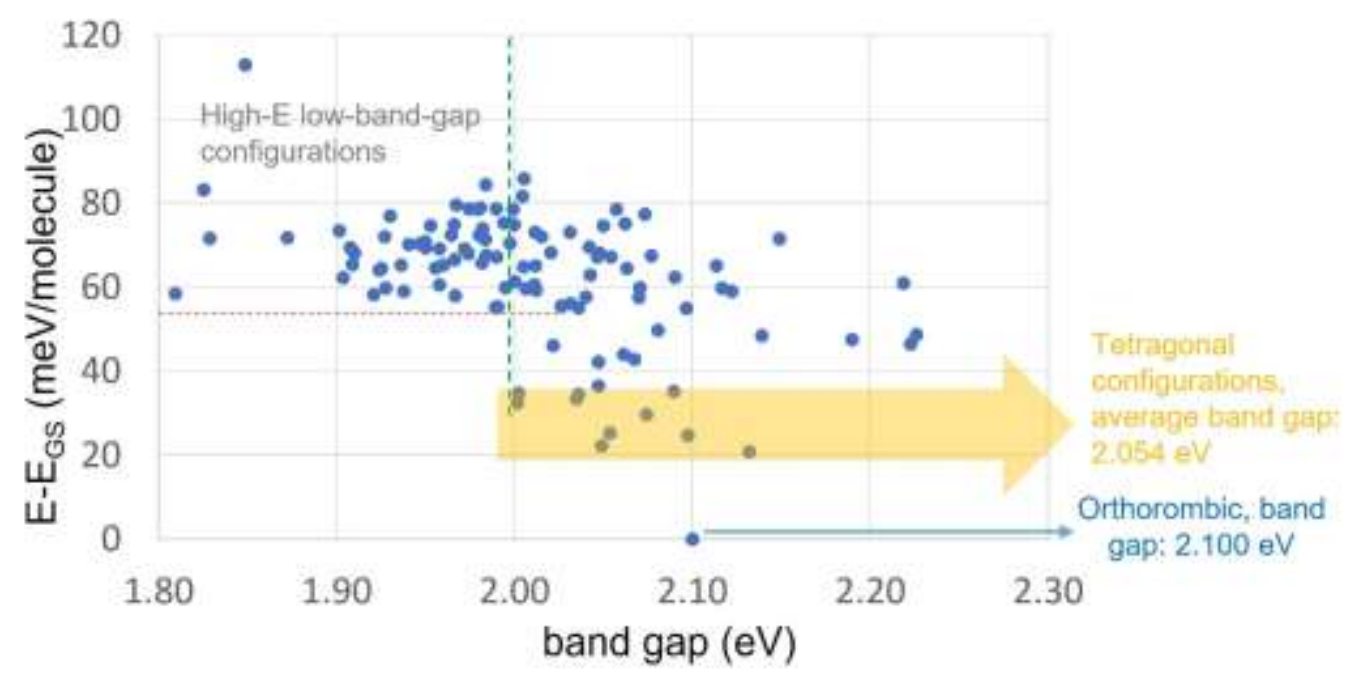

Figure S2. Band gap (horizontal axis) vs DFT energy (vertical axis, structural ground state set to zero) for the $1102 \times 2 \times 2$ supercells (differing by MA configuration) of $\mathrm{MAPbBr}_{3}$ of Fig. 1 of the main text. The dashed lines highlight that all the MA configurations having a band gap lower than $2.0 \mathrm{eV}$ (i.e. on the left of the green line) are high in energy, at least $55 \mathrm{meV} /$ molecule above the ground state (i.e. they all lie above the red line). The arrows pointing to the right indicate the band gap of the lowest-energy structure (orthorhombic phase) and the average band gap of the structures assigned to the tetragonal phase (Fig. 1 of the main text). 


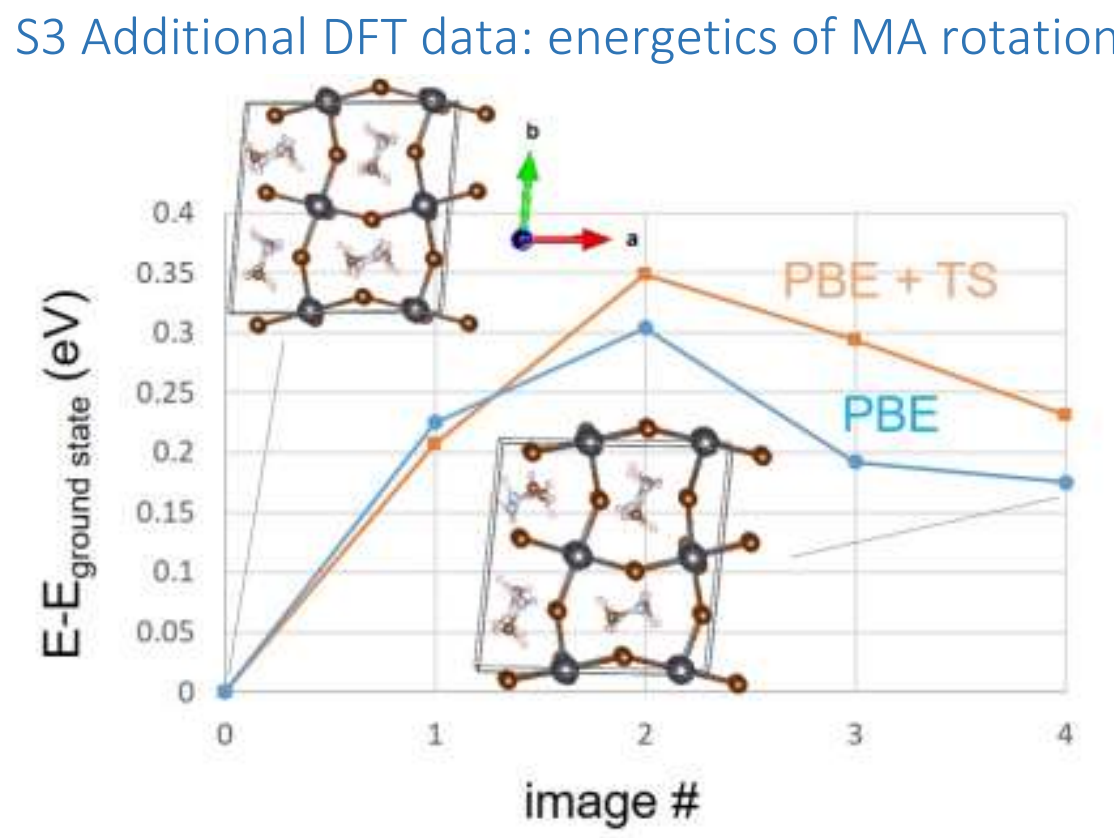

Figure S3. Nudged Elastic Bands results for the $180^{\circ}$ rotation of one MA molecule. The initial and final structures are shown in the insets (the rotated molecules lies in the front, top left unit cell). The energetics along the (minimum-energy) rotation path is shown with and without Tkatchenko-Scheffler dispersion correction.

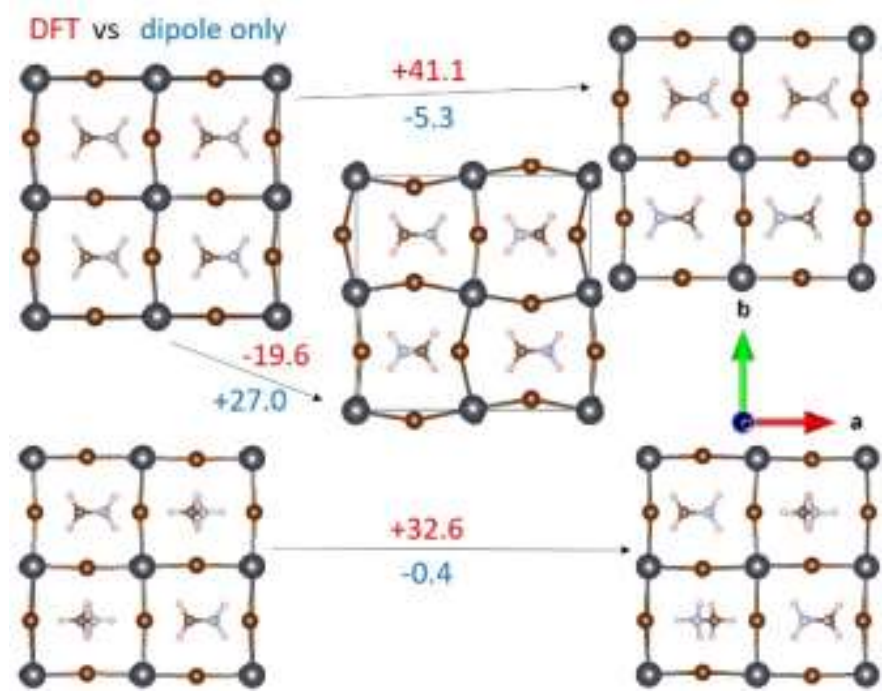

Figure S4. DFT vs dipole-dipole energetics. Here we consider some changes in MA configuration that significantly modify the dipole-dipole interactions while weakly affecting the rest of the structure. We calculated the energy changes accompanying these MA rotations by means of DFT (2x2x1 supercells, 48 atoms; $2 \times 2 \times 4$ k-points net adopted), and with a point charge model in which a +1 and -1 charges are assigned to $\mathrm{C}$ and $\mathrm{N}$ positions, respectively. The DFT and point-charge model energy changes are reported in red and blue, respectively (both in meV/formula unit). Note that, in the point charge model, an infinite 
summation is performed through Ewald summation. Even though the dipole model is a very crude one, these results show that the molecular dipole-dipole energy changes upon varying the MA configuration do not follow the actual, quantum mechanical energy changes. Therefore, it is proven that the dipoledipole interaction among MA molecules is certainly not the main contribution to the energy change upon MA rotation.

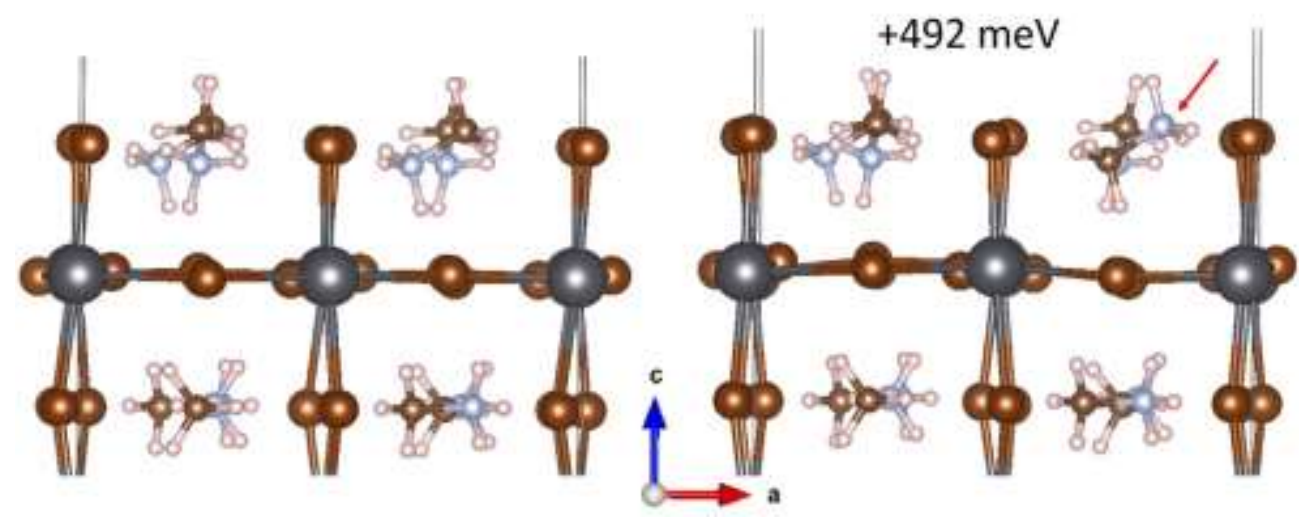

Figure S5 energetics of out-of-surface rotation of the exposed MA molecules on the 001 surface of $\mathrm{MAPbBr}_{3}$. In passing from the structure on the left to the one on the right, the $\mathrm{CN}$ bond of the molecule indicated by a red arrow is rotated by $180^{\circ}$ and kept frozen (all other atoms relaxed). The energy change associated to this rotation is indicated above the right figure. 


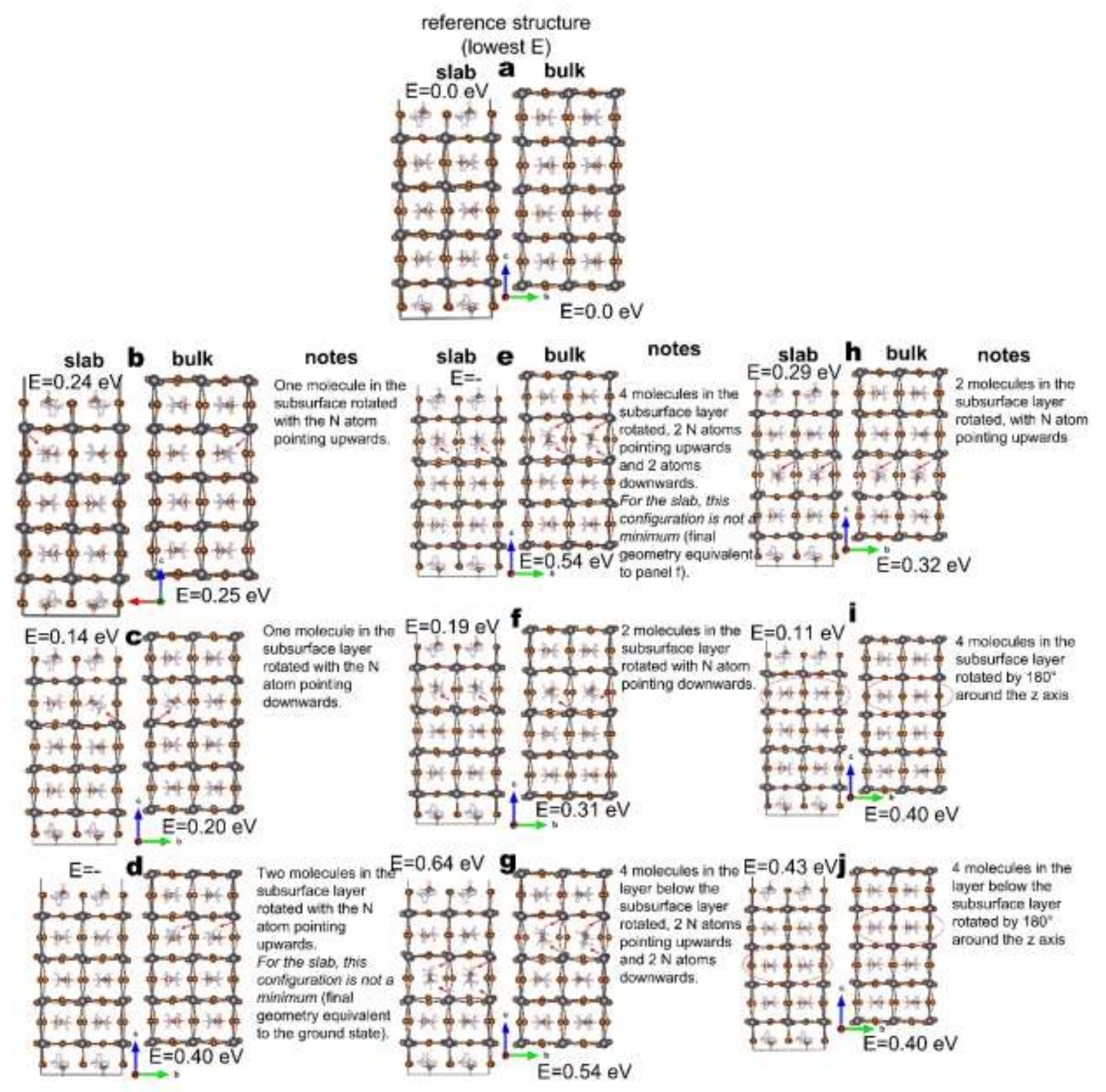

Figure S6. Comparison of (DFT) energetics of MA rotations between slabs and the bulk. In each panel, the results of the rotation of one or more MA molecules are shown (final geometries and energy cost for the rotation). In particular, we built similar cells with vacuum (slab) and without vacuum (bulk), relaxed the geometry after the molecular rotation, and compared the final energy (calculated as the difference with respect to the structural ground state) between the slab and the bulk (see also Sect. 1.1 for the computational details). The rotated molecules in the final geometry are described on the right of each panel and indicated by red arrows/circles. Note that for panels $d$ and e, the slab calculation ended up in a different minimum, despite the starting geometry was the same as in the bulk calculation, further proving the importance of the surface effect. For the bulk configurations, given their 3D periodicity, MA rotations in the subsurface layers and in the layer below are equivalent (e.g. panel i vs j). These results demonstrate 
that the energetics of MA rotation changes significantly between the surfaces and the bulk, thus confirming that the effects shown in Fig. 4 of the main text is not a force field artifact.

S4 Additional DFT data: density of states, charge density plots, and orbital overlap

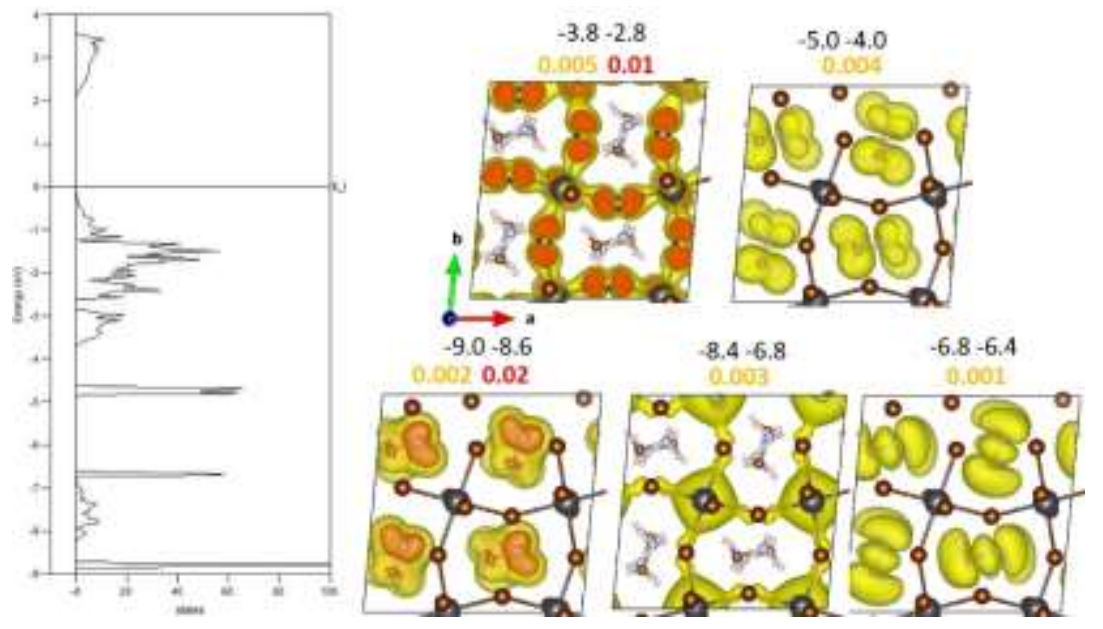

Figure S7. Additional partial charge density plots for the structural ground state of $\mathrm{MAPbBr}_{3}$. Above each charge density plots, the corresponding DOS energy range is reported (black), along with the isosurface values (yellow and red). Note that, differently from the main text, here isosurface values are reported directly in the units used by VASP, namely e/ $\AA^{3}$ multiplied by the unit cell volume $\left(1509 \AA^{3}\right)$. The total DOS is reported for convenience on the left.
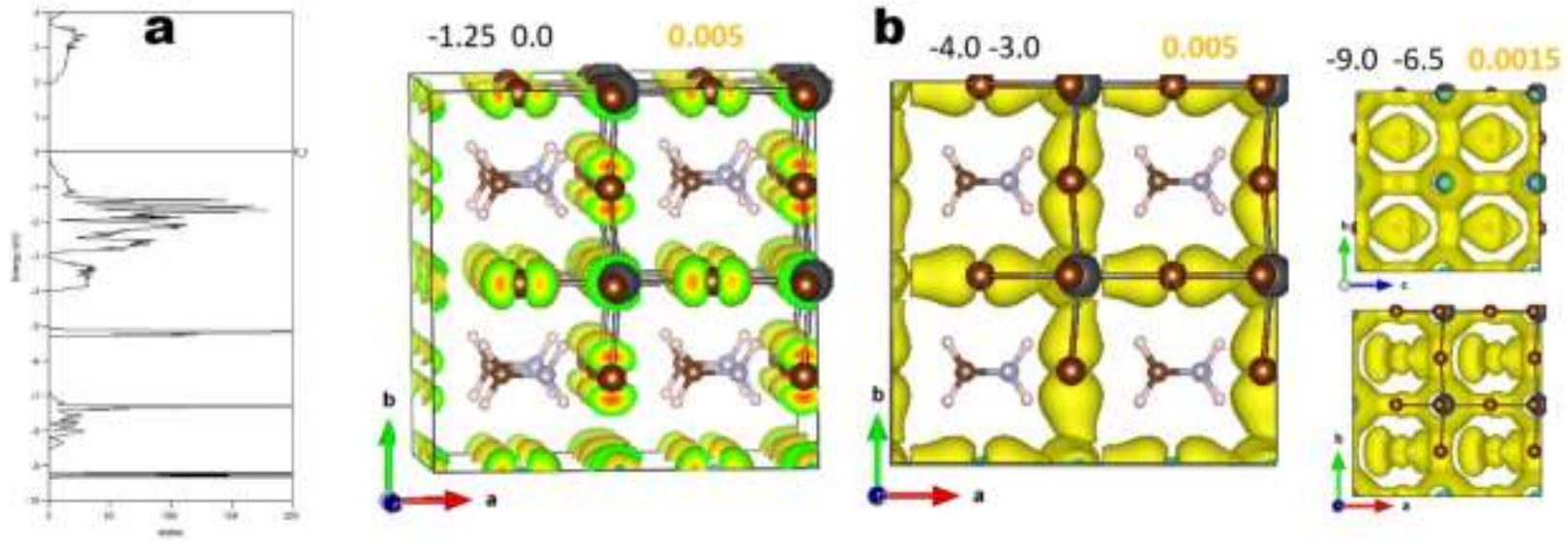

Figure S8. Total DOS and partial charge density plots for the cell (MA configuration) having all MA aligned (configuration IV in Fig. 1 of the main text). Legend as in Fig. S7. 

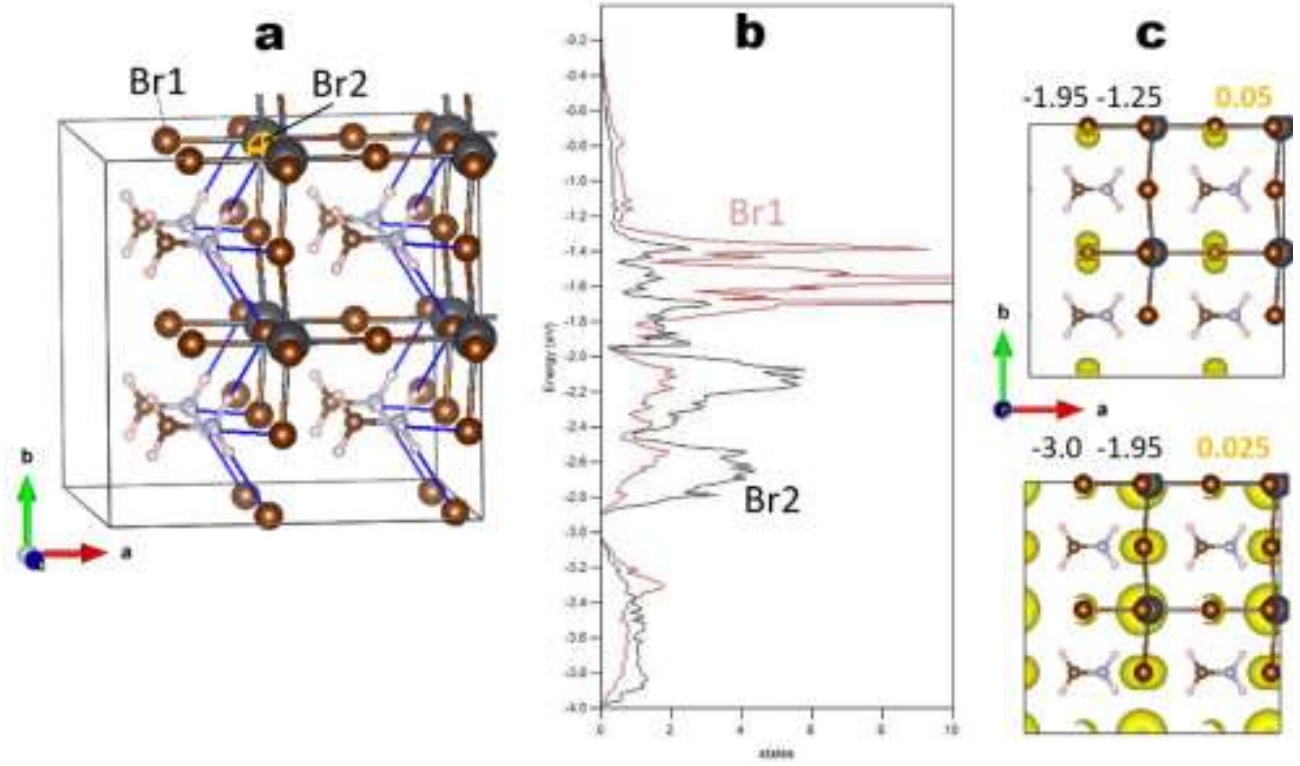

Figure S9. Effect of hydrogen bonds on the energy of Br lone pairs. Since the configuration of Fig. S8 has some $\mathrm{Br}$ atoms accepting several $\mathrm{HBs}$ and other $\mathrm{Br}$ atoms accepting none (HBs indicated in panel a by thin blue lines), it is a good model to study the effect of HBs on the DOS. Panels a and b show that the $\mathrm{Br}$ accepting $\mathrm{HBs}(\mathrm{Br} 2)$ have their p-DOS at lower energy than those not accepting $\mathrm{HBs}(\mathrm{Br} 1)$, thus demonstrating that HBs lower the energy of acceptor lone pairs (as common chemical knowledge would suggest). Panel c (legend as in Fig. S7), shows that the lower and higher energy parts of the band relative to the $\mathrm{Br}$ lone pairs are localized on the $\mathrm{Br}$ atoms that are accepting and that are not accepting strong $\mathrm{HBs}$, respectively.

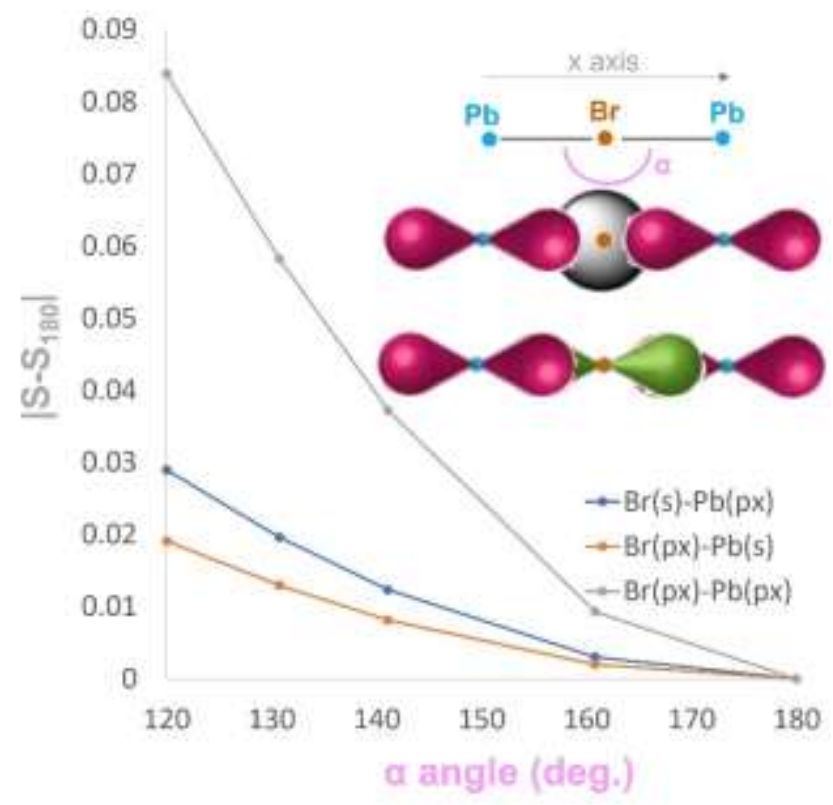


Figure S10. Change in atomic orbital overlap ( $\left.\left|\mathrm{S}-\mathrm{S}_{180}\right|\right)$ with $\mathrm{PbBrPb}$ angle bending $(\alpha)$. Orbitals schematically shown in the top right inset. As discussed in the "Hydrogen bonding and electronic structure" paragraph of the main text, the $\mathrm{Pb}\left(\mathrm{p}_{\mathrm{x}}\right)-\mathrm{Br}\left(\mathrm{p}_{\mathrm{x}}\right)$ overlap (conduction band) decreases faster than the $\mathrm{Pb}(\mathrm{s})-\mathrm{Br}\left(\mathrm{p}_{\mathrm{x}}\right)$ orbital (valence band) when the $\mathrm{PbBrPb}$ angle deviates from 180 deg.. $\mathrm{The} \mathrm{Pb}\left(\mathrm{p}_{\mathrm{x}}\right)-\mathrm{Br}(\mathrm{s})$ orbital overlap change is also reported for comparison, although not directly related to band edge states. The overlap was normalized so that the integral $S_{i i}$ over the whole space gives 1.0. The overlap was calculated with an in-house built fortran90 code which implements the formulas of ref. 17. The ANO-R0 basis $\operatorname{set}^{18}$ (valence orbitals only) was adopted. The value of $\mathrm{Pb}-\mathrm{Br}$ bond length was set to $3.0 \AA$, which is the average of $\mathrm{Pb}-\mathrm{Br}$ bond lengths over the 110 DFT-optimized structures considered in this work (Figs 1,3 of the main text).

\section{S5 Results of TDDFT and large-scale DFT simulations (cp2k)}

Table S2. TDDFT (cp2k code, PBE functional) amplitude and oscillator strength for the electronic transition from the highest-energy occupied state to the lowest-energy empty one, for some of the configurations used in Fig. 1. This electronic transition is the lowest energy one, i.e. the one from the "valence band maximum" to the "conduction band minimum". Note that the geometry is kept frozen at the VASP PBETS estimate, since this geometry is deemed more accurate.

\begin{tabular}{|l|l|l|l|l|}
\hline Config \# & $\begin{array}{l}\text { E-EGS } \\
(\mathrm{meV} / \text { formula } \\
\text { unit) }\end{array}$ & $\begin{array}{l}\text { Excitation } \\
\text { energy (eV) }\end{array}$ & amplitude & $\begin{array}{l}\text { oscillator } \\
\text { strength (a.u.) }\end{array}$ \\
\hline 1 & $\begin{array}{l}\text { a.0 } \\
\text { (ground state) }\end{array}$ & 1.96 & -0.99995 & 0.275 \\
\hline 2 & 46.2 & 2.07 & -0.99991 & 1.263 \\
\hline 3 & 110.0 & 2.00 & -0.99987 & 0.776 \\
\hline 4 & 61.8 & 2.05 & 0.99989 & 0.938 \\
\hline 5 & 69.7 & 1.93 & -0.99991 & 1.11 \\
\hline 6 & 63.4 & 2.17 & 0.99988 & 0.888 \\
\hline
\end{tabular}

a energy taken from more accurate VASP PBE-TS calculation.

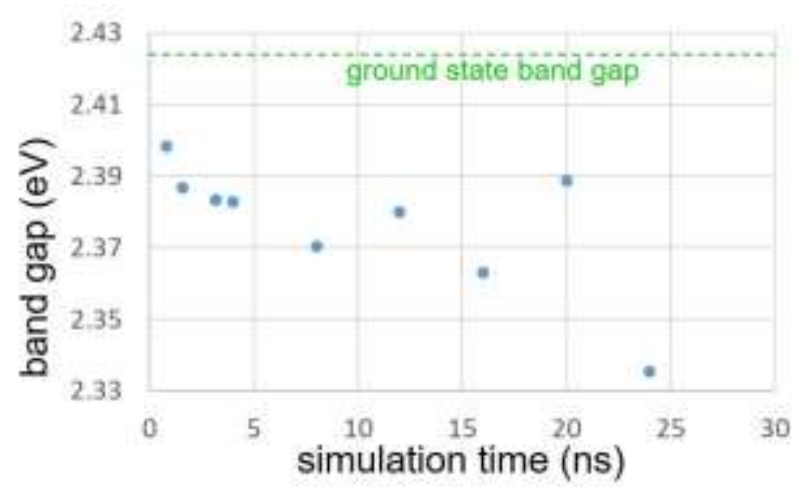

Figure S11. Band gap of the minima explored during an MD run at $\mathrm{T}=300 \mathrm{~K}$ of 3072 atoms of $\mathrm{MAPbBr}_{3}$. The "ground state band gap" refers to the band gap when all the molecules are in the minimum-energy configuration. 


\section{S6 Additional experimental results}

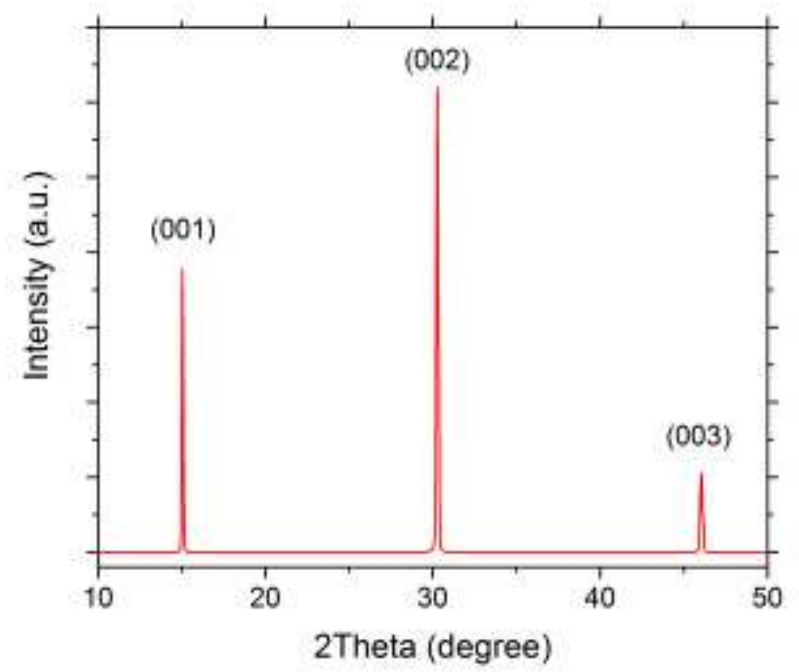

Figure S12 X-ray powder diffraction pattern of the $\mathrm{MAPbBr}_{3}$ sample (grinded single crystal) recorded at room temperature. 


\section{REFERENCES}

${ }^{1}$ Kresse, G. \& Furthmüller, J. Efficiency of ab-initio total energy calculations for metals and semiconductors using a plane-wave basis set. Computational Materials Science 6, 15-50 (1996).

2 Blöchl, P. E. Projector augmented-wave method. Physical Review B 50, 17953-17979 (1994)

${ }^{3}$ https://cms.mpi.univie.ac.at/vasp/vasp/Recommended PAW potentials DFT calculations using vasp $52 . h$ tml

${ }^{4}$ Perdew, J. P., Burke, K. \& Ernzerhof, M. Generalized Gradient Approximation Made Simple. Phys. Rev. Lett. 77, 3865-3868 (1996).

5 Tkatchenko, A. \& Scheffler, M. Accurate Molecular Van Der Waals Interactions from Ground-State Electron Density and Free-Atom Reference Data. Phys. Rev. Lett. 102, 073005 (2009).

${ }^{6}$ Beck, H., Gehrmann, C. \& Egger, D. A. Structure and binding in halide perovskites: Analysis of static and dynamic effects from dispersion-corrected density functional theory.

7 Shi, Dong, et al. "Low trap-state density and long carrier diffusion in organolead trihalide perovskite single crystals." Science 347.6221 (2015): 519-522.

8 G. Henkelman and H. Jónsson "Improved tangent estimate in the nudged elastic band method for finding minimum energy paths and saddle points." J. Chem. Phys. 113, 9901-9904 (2000)

${ }^{9}$ Kühne, T. D. et al. CP2K: An electronic structure and molecular dynamics software package - Quickstep: Efficient and accurate electronic structure calculations. J. Chem. Phys. 152, 194103 (2020).

$10 \mathrm{https}: / /$ manual.cp2k.org/trunk/CP2K_INPUT/MOTION/GEO_OPT.html

11 Hata, T., Giorgi, G., Yamashita, K., Caddeo, C. \& Mattoni, A. Development of a Classical Interatomic Potential for MAPbBr3. J. Phys. Chem. C 121, 3724-3733 (2017).

12 https://lammps.sandia.gov/doc/compute dipole chunk.html

${ }_{13}$ Herzfeld, K. F. Zur Theorie der Reaktionsgeschwindigkeiten in Gasen. Annalen der Physik 364.15, 635667 (1919)

${ }^{14}$ Vineyard, G. H. "Frequency factors and isotope effects in solid state rate processes." Journal of Physics and Chemistry of Solids 1-2, 121-127 (1957).

${ }^{15}$ Eyring, H. "The activated complex in chemical reactions." The Journal of Chemical Physics 3(2), 107115 (1935).

16 Leguy, A. M. A. et al. Dynamic disorder, phonon lifetimes, and the assignment of modes to the vibrational spectra of methylammonium lead halide perovskites Phys. Chem. Chem. Phys., 18, 2705127066 (2016).

${ }^{17}$ Cook, David B. Ab initio valence calculations in chemistry. Butterworth-Heinemann, 2013

18 Zobel, J.P., Widmark, P.O., \& Veryazov, V. "The ANO-R Basis Set” J. Chem. Theory Comput. 2020, 16, 1, 278-294 (2020). 